\title{
Belphégor
}

\section{Fabrice Leroy, Sfar So Far : Identity, History, Fantasy, and Mimesis in Joann Sfar's Graphic}

\section{Chris Reyns-Chikuma}

\section{(2) OpenEdition}

1 Journals

\section{Édition électronique}

URL : http://journals.openedition.org/belphegor/544

DOI : 10.4000/belphegor.544

ISSN : 1499-7185

Éditeur

LPCM

\section{Référence électronique}

Chris Reyns-Chikuma, "Fabrice Leroy, Sfar So Far : Identity, History, Fantasy, and Mimesis in Joann Sfar's Graphic », Belphégor [En ligne], 13-1 | 2015, mis en ligne le 09 mai 2015, consulté le 22 septembre 2020. URL : http://journals.openedition.org/belphegor/544; DOI : https://doi.org/10.4000/belphegor. 544

Ce document a été généré automatiquement le 22 septembre 2020.

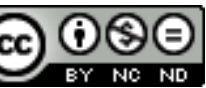

Belphégor est mis à disposition selon les termes de la Licence Creative Commons Attribution - Pas d'Utilisation Commerciale - Pas de Modification 4.0 International. 
Fabrice Leroy, Sfar So Far : Identity, History, Fantasy, and Mimesis in Joann Sfar's Graphic

Chris Reyns-Chikuma

\section{RÉFÉRENCE}

Fabrice Leroy, Sfar So Far : Identity, History, Fantasy, and Mimesis in Joann Sfar's Graphic Novels. Leuven : Leuven University Press LUP, 2014. ISBN : 9789462700062 
1 Ceci est indubitablement un très bon et très beau livre. Il est bon dans le sens où il est bien écrit, bien argumenté, bien structuré, et riche, mais aussi dans le sens où il est beau car il est présenté dans une édition de qualité sur papier glacé, avec de superbes reproductions de pages ou de cases, en noir et blanc dans le texte et en couleur en fin de volume. C'est le second volume d'une toute nouvelle collection (2014) créée par les éditions de LUP (Leuven University Press). Comme son titre l'indique, "Studies in European Comics and Graphic novels », la collection a pour but de se limiter à la bande dessinée européenne. Elle cherche d'autre part à embrasser les multiples traditions

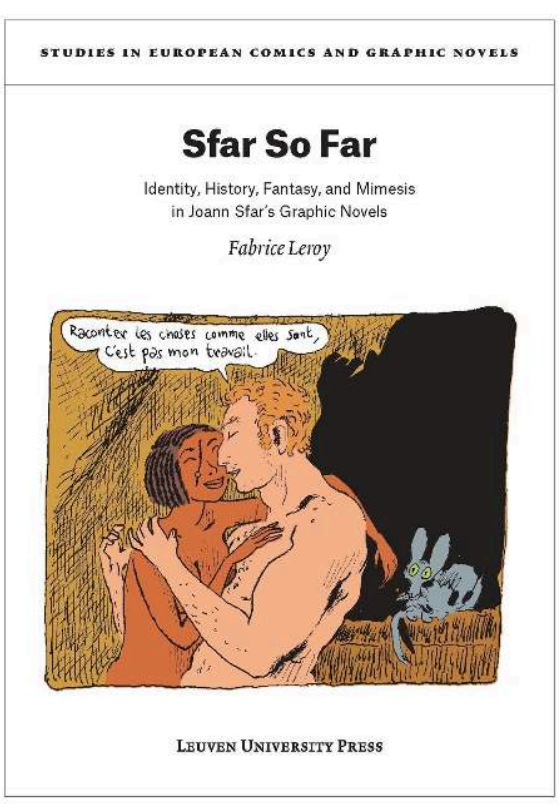
tant régionales (comme la flamande par exemple) que nationales (italienne, espagnole ...). À travers son choix de la langue anglaise la collection s'adresse à un large public qui n'est pas nécessairement familier du sujet. Sfar est encore peu traduit en anglais (ce qui est le cas de très nombreux romans graphiques francophones, contrairement aux Tintin, Astérix et $\mathrm{C}^{\mathrm{ie}}$ ), mais les quelques dix œuvres dont parle Leroy le sont toutes et un public non-francophone, international, pourra donc les lire.

Cette étude sera d'autant plus appréciée que c'est la première monographie sur cet auteur phare (si j'ose ajouter un jeu de mots à celui du titre du livre) de la bande dessinée, et au-delà, de la culture française contemporaine, puisqu'il a aussi produit des romans et des films, depuis presque deux décennies. Il a publié plus de cinquante ouvrages, seul ou en collaboration, unique (one shot) ou dans des séries avec d'autres auteurs clés - Trondheim, Guibert, Blain ....- ou débutants - Tanquerelle -. Il s'est montré à l'aise dans les genres les plus divers (SF, fantastique, heroic fantasy, réalisme magique), mêlant des tons les plus inattendus et faisant des références directes ou indirectes des plus riches et subtiles à l'histoire et au contexte postcolonial et multiculturel français.

Le titre (« so far » - « jusqu'à maintenant ») met pourtant bien en évidence que l'étude porte sur un corpus limité. Tout d'abord, sfar étant encore relativement jeune (il est né en 1971) et sa carrière est loin d'être finie (ce qui est encore souligné dans la conclusion, p. 225). Au-delà, le corpus de "Sfar So Far » est aussi limité à une dizaine d'œuvres, surtout des œuvres produites seul qui appartiennent plus au roman graphique qu'à la bande dessinée. Cette étude se concentre en fait sur une période « limitée » de 2000 à 2014 (Pascin, Klezmer, Le Chat du rabbin, Chagall en Russie, Les Lumières de la France ...). Elle ignore donc la première période faite d'expérimentations diverses souvent en collaboration avec d'autres succinctement présentée dans l'introduction peut-être trop succinctement pour un public qui ne connaît pas Sfar, ce qui est probablement le cas de la majorité des Anglo-saxons, y compris dans le milieu des Comics Studies. De fait, à quelques exceptions près (Miller, Frey, McKinney, Beaty, d'ailleurs cités dans l'étude - voir biblio, pp.276-84), ce champ de recherche est 
souvent enfermé dans son univers anglophone tant de Superhéros que de graphic novels anglo-saxons, il est vrai, déjà si riche.

4 L'intérêt de cette étude se trouve tant dans les micro-analyses que dans la contextualisation de la production de Sfar. Les micro-analyses mettent en évidence l'extrême subtilité de l'œuvre sfarienne, et par conséquent aussi du média/art de la bande dessinée en général. Comme pour Spiegelman et Maus en particulier, c'est ce genre d'études qui manifeste le fait que ce média n'est pas un art mineur et pas seulement pour mineur/e/s, mais bien un art/média mûr. Ainsi Leroy rappelle que Sfar a fait des études à la fois de philosophie et d'art avec un mémoire intitulé Le complexe du golem ou le peintre juif à la figure humaine (1993). Il manifeste le fait que les références aux peintures précises comme celles que l'on découvre dans l'épisode de la carcasse achetée dans Pascin se référent aux canevas de Soutine, ou dans les scène antisémites au bordel qui font écho aux dessins de Pascin pour la revue Simplicissimus. Leroy établit donc un «Metaphoric reading of some episodes» entre par exemple «boucherie/war/pogrom/ holocaust » (p. 42) et une riche intertextualité tant avec le « Homer's Ulysse driving a stake into Polyphemous's eye" qu'avec le "David defeating Goliath and a similar episode in Pratt's Suite Caribéenne » (p. 47).

Une table des matières donne six parties, une introduction, quatre chapitres et une conclusion, suivies de notes détaillées (pp. 253-72) et d'une bibliographie des plus éclectiques (Homère, Le Clézio, Lovecraft, ... pp. 273-85). Sont visibles, dans la section des références bibliographiques - qui comprend tant des textes de fiction, y compris des bandes dessinées, que des essais, des travaux sur le fantastique (Bessière, Todorov...) - des références sur la bande dessinée, théoriques (Groensteen) et historiques (Kunzle, Ann Miller...), et des travaux historiques centrés sur les problématiques abordées (comme sur l'esclavage, le colonialisme, l'antisémitisme ou la peinture), y compris des textes originalement en français, traduit en anglais (Winock). L'étude de Leroy, professeur aux États-Unis (Louisiane) est plus en ligne avec la tradition anglo-saxonne des cultural studies (p. 25) qu'avec les tendances européennes et plus encore françaises de la sémiotique. De manière surprenante pour une étude très anglo-saxonne, Sfar so Far ne contient pas d'index. Mais ceci est sans doute perçu comme inutile dans la mesure où le texte est clairement divisé en de nombreuses unités et sous-unités qui rendent toute recherche de terme, thème ou œuvre, facile, et où il contient des notes en fin de volume très claires et fournies. On notera encore que Leroy n'utilise pas de jargon inutile. Si parfois il emploie un vocabulaire technique, celui-ci est toujours approprié, nécessaire et précis pour mieux comprendre la subtilité de la représentation (analepsis, focalisation interne et externe, par exemple).

6 Sfar So Far se termine aussi par un entretien (en anglais ou traduit ?) de seize pages qui redonne la parole aux deux auteurs finissant ainsi sur une note plus personnelle et tout aussi intéressante, confirmant pour celui qui ne serait pas encore convaincu que Sfar est un « nouveau » maître du $9^{e}$ art qu'il faudra suivre et Leroy un nouveau spécialiste avec qui il faudra compter (pp. 236-52). 